\title{
2x4 Hybrid MZI-MMI Configuration with MMI Phase-shifters as a High-speed Optical Switch with Low Power Consumption
}

\author{
Boris B Niraula ${ }^{1}$ and Conrad Rizal ${ }^{2, *}$ \\ ${ }^{1}$ SeedNanoTech and Consulting, Brampton, ON, Canada, L6Y3J6 (boris.niraula@seednanotech.com) \\ ${ }^{2}$ Department of Electrical Engineering \& Computer Engineering, The University of British Columbia, \\ Vancouver, BC, Canada; criza@ece.ubc.ca \\ ${ }^{*}$ Correspondence: BN(boris.niraula@seednanotech.com)
}

\begin{abstract}
This paper reports $2 \times 4$ hybrid Mach-zehnder interferometer (MZI) - multi-mode interferometers (MMI) based compact thermo-optical switch consisting of slab waveguides on silicon-on-insulator, SOI, platform. The device consists of two identical MMIs, each of $6 \mu \mathrm{m}$ wide and $140 \mu \mathrm{m}$ long connected with two phase shifters MMIs each with $2 \mu \mathrm{m}$ wide and $8 \mu \mathrm{m}$ long and linear tappers each $4 \mu \mathrm{m}$ long, connected at both ends of the MMIs to minimize the power coupling loss. The loss for linear taper is found to be below $0.02 \mathrm{~dB}$. The footprint of the whole device is $6 \mu \mathrm{m}$ $\times 324 \mu \mathrm{m}$. This structure is based on a unique multimode region shape, which leads optical switch to have less coupling loss and reduced cross-talk. The average thermo-optical switching power consumption is $1.4 \mathrm{~mW}$, the excess losses are $0.8 \mathrm{~dB}$, and the imbalances are $0.1 \mathrm{~dB}$. Aluminum is used as a heating pad, and a trench is created around this pad to prevent from spreading of heat and reduce power loss. Our new heating method has advantages of compact size and ease of fabrication with the current CMOS technology.
\end{abstract}

Keywords: Multimode Interferometer, thermo-optical Switch, phase shifter, Silicon on Insulator, SOI technology, bio-sensor

\section{Nomenclatures}

\section{Abbreviations}

SOI Silicon on Insulator

MMI Multimode Interferometer

CMOS Complementary Metal Oxide Semiconductor

\section{Symbols}

$\beta \quad$ Propagation constant

$\alpha$ Propagation constant

$\lambda$ Operation optical wavelength

$\phi \quad$ Phase shift induced due to the thermo-heater

$\Delta \mathrm{L}_{\pi}$ Path length difference

\section{Introduction}

\footnotetext{
* Current address: York University, GEM Systems Inc, Markham, ON, L3R 5H6, Canada (conrad.rizal@gemsys.ca)
} 
The consistent and continuous demand/growth of high-speed internet with a higher volume of data processing prompts the need for high-speed non-blocking optical switches with less sensitivity to polarization and operational wavelength [1]. These functionalities need to accompany with low power consumption, low loss, small device footprint, and a higher number of ports, full operational bandwidth that depends on the number of output ports, and low production cost.

The silicon-on-insulator (SOI) material platform supports the design of compact wave-guiding devices with reasonable fabrication tolerance and cost and has attracted renewed research interest. Comparison of technical characteristics of presently operational and proposed optical switches based on MMI waveguides is given elsewhere [2] and is not described here. Presently operational as well as proposed optical switches offer transmission of data of up to $40 \mathrm{GBps}$ and the average operating speed of up to $10 \mathrm{GHz}$ : the demand for high-speed internet and the vast amount of data transmission calls for a new generation of photonic switches.

Interaction of light with photonic waveguides results in interesting interference patterns, and it is this interference patterns that are utilized in realizing optical switches of MMI configuration as these patterns allow efficient de-coupling and coupling of the light beam as it propagates through waveguides. Two widely used photonic waveguiding configurations that are commonly used in realizing switching, modulating and filtering functionalities are directional coupler based Machzehnder interferometry (MZI) [3] and multimodal coupler based interferometry (MMI) [4].

MZI based waveguiding configurations consists of directional couplers (DCs), tappers and optical wires of different dimensions and geometry [5]. These operate over wide /broad optical bandwidth, and they show environmental stability. These, however, suffer from low fabrication error/tolerance, higher access and bending loss and high-power consumption, and show wavelength dependent transmission spectra and quality factor.

Unlike MZIs, MMI-based wave-guiding configurations consist of slab-like photonics structure with connectors and ring resonators of different shape and geometry [4]. These couplers work on the principle of self-imaging effect [6] and function as better coupler and de-coupler of the light beam as opposed to directional couplers. MMI based couplers/splitters as switching configurations are expected to offer many additional advantages over MZI based switching configurations as they show well-defined splitting/coupling ratios, lower excess loss, low polarization sensitivity, better dimensional tolerance and ease of fabrication, wavelength insensitive transmission spectra and wavelength independent quality factor and support many ports.

Si photonics extensively employ micro-ring resonators (MRR), which usually consists of a wire-like circular waveguide-coupled with one or two straight waveguides [5,7]. The advantage of incorporating MRR into MMI configuration is that the resulting device produces transmission spectra with narrow spectral width line and high-quality factor (Q)[8]. MRR can also be coupled with both the DCs and MMIs to achieve coupling/splitting between the ring and the bus waveguides. When coupled with directional couplers the MRR resonators produce lower insertion loss, which is useful in realizing very high $Q$, usually needed for sensing and switching applications, and for studying non-linear optical effects. The problem of integrating MRR with MMIs or DCs lies in difficulties of fabrication - the fabrication requirement of DC-coupled MRRs is strict, which is the main drawback in achieving a standard device.

Even though the operating bandwidth of the MRR increases with the coupling of MMI, the configuration suffers from another unwanted drawback of relatively large insertion loss and reducing the $\mathrm{Q}$ factor significantly. These drawbacks though compatible with high-speed signal processing and optical delay lines; they are not desirable for high-speed optical switching and sensing operations.

A large number of thermo-optical switches based on MMI configuration and plasmonic circuitry have been proposed, designed and demonstrated [9]. These came with a variety of device geometry, functionalities, and performance characteristics. The simplest of these was proposed, designed, and demonstrated earlier by Zinke et al. in 1997 [10] and recently by Ota et al. [11] and Oulton et al. [12]. 
The device can be classified as a hybrid of MZI and MMIs, with cross talk of about $-35 \mathrm{~dB}$ and power loss of about $0.2 \mathrm{~dB}$, but an inertion loss of around 3.5 to $8.0 \mathrm{~dB}[12,13]$ and waveguide propagation loss and the modulation loss from the "bar" state to the "cross" state 0.8 and $1 \mathrm{~dB}$, respectively.

Xie et al. [14] designed and demonstrated low power consuming, high-speed polarization independent polymer-based hybrid MMI-MZI switching architecture that showed wide operational bandwidth of $80 \mathrm{~nm}$, low cross-talk and power consumption of the heating switch of around $10 \mathrm{~mW}$.

In the recent decade, many research groups have designed and demonstrated the possibility of realizing multi-portal optical switch of $\mathrm{N} \times \mathrm{N}$ kind using hybrid MMI-MZI architecture consisting of $1 \times 2$ and $2 \times 2$ switching elements coupled with the micro-ring resonator or directional couplers [15]

Coupling of MZI based switching platform with MMI leads to small and compact device sizes with substantial benefits such as low excess loss, well-defined splitting and coupling ratios, fabrication friendly, multiple port support and polarization independent switching device [14]. In these switching platforms, MZI is connected to multiple MMIs using an array of phase shifters as proposed by Lagalliet al. and Morrissey et al. [16,17]. These devices are also widely known as generalized MZIs, and these offer the advantages of $\mathrm{N}$ independent switching states, supporting multiple ports and multiple switching functions. Unfortunately, these devices act as blocking switches.

To overcome this problem Li et al. [18] proposed and demonstrated 4/4 non-blocking switch by cascading four $2 \times 2 \mathrm{MZI}$ on both sides of an MZIs and by doing so they produced a balanced waveguide configuration. In total, this device includes 5 MZI switching elements. The device, however, suffers from higher insertion loss and a more non-uniform power splitting ratio.

We have proposed, designed, and demonstrated/characterized a $2 \times 4$ hybrid MZI-MMI configuration in which two separate slabs of $140 \mu \mathrm{m} \times 6 \mu \mathrm{m}$ each are connected via two MMI based phase shifters, each with $2 \mu \mathrm{m} \times 8 \mu \mathrm{m}$ waveguides, giving a compact, high-speed optical switch of $324 \mu \mathrm{m} \times 6 \mu \mathrm{m}$ device footprint. The four MMIs, in this case, are connected using tapered waveguides. The device consists of 2 input ports and four polarization independent, non-blocking ports.

Similarly to Ref [19], a heating element is incorporated in one of the phase shifter arms to realize thermo-optical switching and a specially designed heating pad is used to heat this arm. It consists of thin $\mathrm{Al}$ pad and can be placed on the phase shifting arm directly. Literature search in this area suggested that such a simple, yet others have not demonstrated a robust and compact device.

\section{Theoretical Foundation}

The operation principle of MMI is based on self-imaging theory and total internal reflection [6,20-29]. Making use of the self-imaging principle involves exciting a set of guided modes in the multimode section by a fundamental mode from one of the input ports. A perfect self-imaging involves reproduction of an input mode profile in single or multiple images at periodic intervals along the propagation direction of the multimode section as shown in Fig $\mathbf{1}(\mathbf{a}, \mathbf{b})$. The propagation constants, in this case, is given by: $\Delta \beta_{m}=\frac{m(m+2) \pi}{3 L_{\pi}}$, where, $\Delta \beta_{m}$ is the propagation difference between two consecutive modes, and $\mathrm{L}_{\pi}$ is the beat length/coupling length between lowest order modes and is given by [6]: $\mathrm{L} \pi=\frac{\pi}{\left(\beta_{0}-\beta_{1}\right)}$, where, $\beta_{0}$ and $\beta_{1}$ denote the propagation constants of fundamentalthe and first mode, respectively.

As explained by Ulrich [29] and Soldano [25], self-imaging is achieved when the input field is reproduced as single or multiple images along the dimension of the MMI slab at a periodic interval. For an input electromagnetic field of $\Psi(\mathrm{y}, 0)=\sum_{v=0}^{M-1} c_{v} \Psi_{v}(\mathrm{y})$ where $\Psi_{v}(y)$ is the normalized mode profile of the $\mathrm{v}^{\text {th }}$ mode and $c_{v}$ is the field excitation coefficient and is given by the overlap integral, $\int \Psi(\mathrm{y}, 0) \Psi_{v}^{*}(\mathrm{y}) d y / \int\left|\Psi_{v}{ }^{2}(x)\right|^{2} d y$ where ${ }^{*}$ denotes the complex conjugate. The field profile at $\Psi(\mathrm{y}, \mathrm{z}=\mathrm{L})$ is given as, $\Psi(\mathrm{y}, \mathrm{z}=\mathrm{L})=\sum_{v=0}^{M-1} c_{v} \Psi_{v}(\mathrm{y}) e^{-j \beta_{v} L}$, where $\Psi(\mathrm{y}, 0)$ is the field profile at $\mathrm{y}=\mathrm{L}$, i.e., at the output port of the MMI. The output profile at $y=L$ is a replication of the field profile $\Psi(y, 0)$ at $y=0$. The $\beta_{v}$ is the propagation constant of the $v^{\text {th }}$ mode [30]. The replication of the input field profile at regular interval along the la ength, LMmI, is given by the following conditions: 
a) For a single image, $\mathrm{Lmmi}=\mathrm{P}(3 \mathrm{~L} \pi(\lambda))$, where $\mathrm{P}$ is the input field power, and it is equal to 0,1 , 2 , etc.

b) For multiple images, $\mathrm{LmmI}_{\mathrm{I}} \mathrm{P}\left(3 \mathrm{~L}_{\pi}(\lambda)\right) / 2$, where $\mathrm{P}=0,1$, 2, etc.

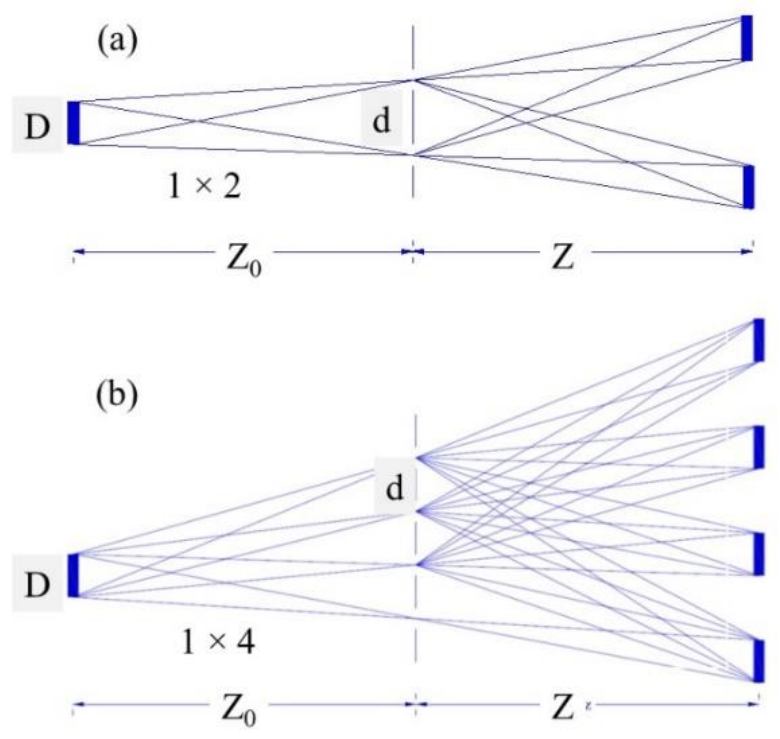

Figure 1. Schematic diagram of the formation of Fresnel images when the light is confined in media: (a) formation of $1 \times 2$ images and (b) formation of $1 \times 4$ images.

These replicated images of the input power can be used to achieve either single or multiple output ports at the exit of the device. For example, when $\mathrm{LmMI}=2 \mathrm{P}\left(3 \mathrm{~L}_{\pi}\right)$, the field reproduced at the output port at Lmm is in phase with respect to the field at the entrance port, meaning the coupler at the exit is at bar state. When Lмmi $=(2 \mathrm{P}+1) 3 \mathrm{~L}_{\pi}$, then the image reproduced is in the antiphase with respect to the field at the entrance port, meaning the coupler at the exit is in cross state. When LmmI $=(P+1 / 2)$ $3 \mathrm{~L} \pi$, the field at the exit port is the linear combination of its input field and its mirror image and the device at the exit is in the $3-\mathrm{dB}$ state.

Since the light beam is confined in a media because of obeying total multiple Fresnel reflections conditions, the beam undergoes multiple periodically repeating self-interference patterns. For instance, self-generation of two interference patterns with equal output powers can be achieved using the relationship as [6]: $\mathrm{LMMI}_{\mathrm{M}} \mathrm{M} \times 3 \times \mathrm{L}_{\pi} / \mathrm{N}$, where $\mathrm{M}$ and $\mathrm{N}$ are any positive integers without a common divisor, $\mathrm{N}$ are the number of self-replicating interferences, and $\mathrm{M}$ defines device length with various $\mathrm{N}$.

The relationship suggests that a compact device is obtained for $M=1$. For the MMI width $W_{e}$ and effective refractive index, Neff and when excited from either input or output ports, the $\mathrm{L}_{\pi}$ can also be given by $4 \times \mathrm{N}_{\text {eff }} \times \mathrm{We}_{\mathrm{e}} /(3 \times \lambda)$. That means, $L_{\pi}$ is related to the effective width, $W_{e}$, and wavelength, $\lambda$, and $N_{\text {eff. }}$ For a lateral wave guided structure, the $W_{e}$ is related to the geometric width of the device as [25]: $W_{e}=W_{M M I}+\frac{\lambda}{\pi}\left(\frac{N_{c}}{N_{e f f}}\right)^{2} \frac{1}{\sqrt{\left(N_{\left.e f f^{2}+N_{c l d}{ }^{2}\right)}\right.}}$, where, $N_{\text {cld }}$ is the effective refractive index of a cladding layer. When the MMI of width We and core refractive index $\mathrm{N}_{\text {eff }}$ is excited from one of the input ports, the beat length can be expressed as: $L_{\pi}=\frac{4 N_{e f f}(\lambda) W_{e}{ }^{2}}{3 \times \lambda}$, where the phase difference between the even and odd modes at the end of the multi-mode region is $\pi$. At one beat length and a half, the MMI produces a pair of identical images, making the waveguide a $3 \mathrm{~dB}$ coupler. Electrical fields inside the MMIs are given as: $E(x, z)=e^{(-j k z)} \sum_{m=1}^{M} E_{m} e^{\left(j \frac{m^{2} \pi}{4 \Lambda} z\right)} \sin \left(\frac{m \pi}{W_{M M I}} x\right)$, where $M$ is the total number of modes that the chosen MMI can support, and $m=0,1,2, \ldots, \wedge=\mathrm{nW}^{2} / \lambda$ ( $\mathrm{n}=$ refractive index, $\mathrm{W}_{\mathrm{MMI}}$ is the MMI width, and $\lambda$ is the operating wavelength) [21]. 
The geometrical characteristics of the device (access waveguide and MMIs) are given in section 3. These have been estimated and chosen using device performance simulations tool that employed transfer matrix method [2, 39], whereas modal propagation method was employed for the optimization of the physical parameters of its components. The given parameters were chosen as these showed strong confinements of the optical field inside the device.

\section{Design, simulation, and optimization}

\subsection{Multimode Interferometers}

The design of the MMIs was based on SOI technology, and the schematic diagram of the proposed MMI device is given in Fig 2. The device consists of two identical MMIs of $140 \mu \mathrm{m} \times 6 \mu \mathrm{m}$ connected via two smaller phase shifter MMIs each with $2 \mu \mathrm{m} \times 8 \mu \mathrm{m}$. The $\mathrm{MMI}_{1}$, in this case, is designed to act as a power splitter whereas $\mathrm{MMI}_{2}$ is designed as a power coupler. $\mathrm{MMI}_{3}$ and $\mathrm{MMI}_{4}$ are used as connectors between $\mathrm{MMI}_{1}$ and $\mathrm{MMI}_{2}$ and these also act as phase shifters as the heating element is incorporated in one of these. As shown in Fig. 1, the device is designed to act as a multiple channel waveguide with four output tapered ports, and 2 input tapered ports.

As shown in the inset of Fig 2, a standard silicon slab of the refractive index of 3.48, the thickness of $h_{\text {core }}=220 \mathrm{~nm}$ and access waveguide width of $\mathrm{W}_{\mathrm{a}}=0.5 \mu \mathrm{m}$ at the input side was chosen to achieve single mode operation. These were obtained using modal analysis method and confirmed with FDTD mode solver.

Table 1 Geometrical characteristics of the device: access waveguide and MMIs.

\begin{tabular}{c|c|c|c|c}
\hline & \multicolumn{3}{|c}{ Geometrical and physical characteristics of waveguides } \\
\hline \multicolumn{2}{|c|}{ Access waveguide } & \multicolumn{2}{c}{ MMI } \\
\hline Material & Ref. Index, $\mathbf{n} @ \boldsymbol{\lambda}=\mathbf{1 5 5 0} \mathbf{~ n m}$ & Dimension $(\mu \mathrm{m})$ & Parameters & Dimension $(\mu \mathrm{m})$ \\
\hline Substrate, $\mathrm{Si}$ & 3.45 & 1000 & Thickness, $\mathrm{t}$ & 0.22 \\
\hline $\begin{array}{c}\text { Lower cladding, } \\
\mathrm{SiO} \text {, }\end{array}$ & 1.45 & 0.50 & Length, L & 140 \\
\hline Core, $\mathrm{Si}$ & 3.45 & & & \\
\hline Upper cladding, $\mathrm{Air}$ & 1.0000005 & - & Width, $\mathrm{W}$ & 6 \\
\hline
\end{tabular}

The access waveguide is tapered from $\mathrm{W}_{\mathrm{a}}=0.5 \mu \mathrm{m}$ to a width, $\mathrm{W}_{\mathrm{tp}}=0.8 \mu \mathrm{m}$. Air, with a refractive index of 1.0000005 is considered as the upper cladding whereas $\mathrm{SiO}_{2}$, with a refractive index of 1.45 as the lower cladding. The geometrical parameters of the $\mathrm{MMI}_{1}$ and $\mathrm{MMI}_{2}$ are estimated to be: thickness $=0.22 \mathrm{~nm}$, length $=140 \mu \mathrm{m}$ and width $=6 \mu \mathrm{m}$. The refractive index of the silicon core is $\mathrm{nsi}$ $=3.48$. These device characteristics were estimated assuming that the optical signal used is TE polarized (s-polarized) and that its functional wavelength, $\lambda$ is $1550 \mathrm{~nm}$. The length of the $\mathrm{MMI}_{3}$ and $\mathrm{MMI}_{4}$, used as phase shifters, is $\mathrm{L}=8 \mu \mathrm{m}$ each (see Fig. 3). The access waveguide is tapered to improve device performance. The parameters used in the design are listed in Table 1. 


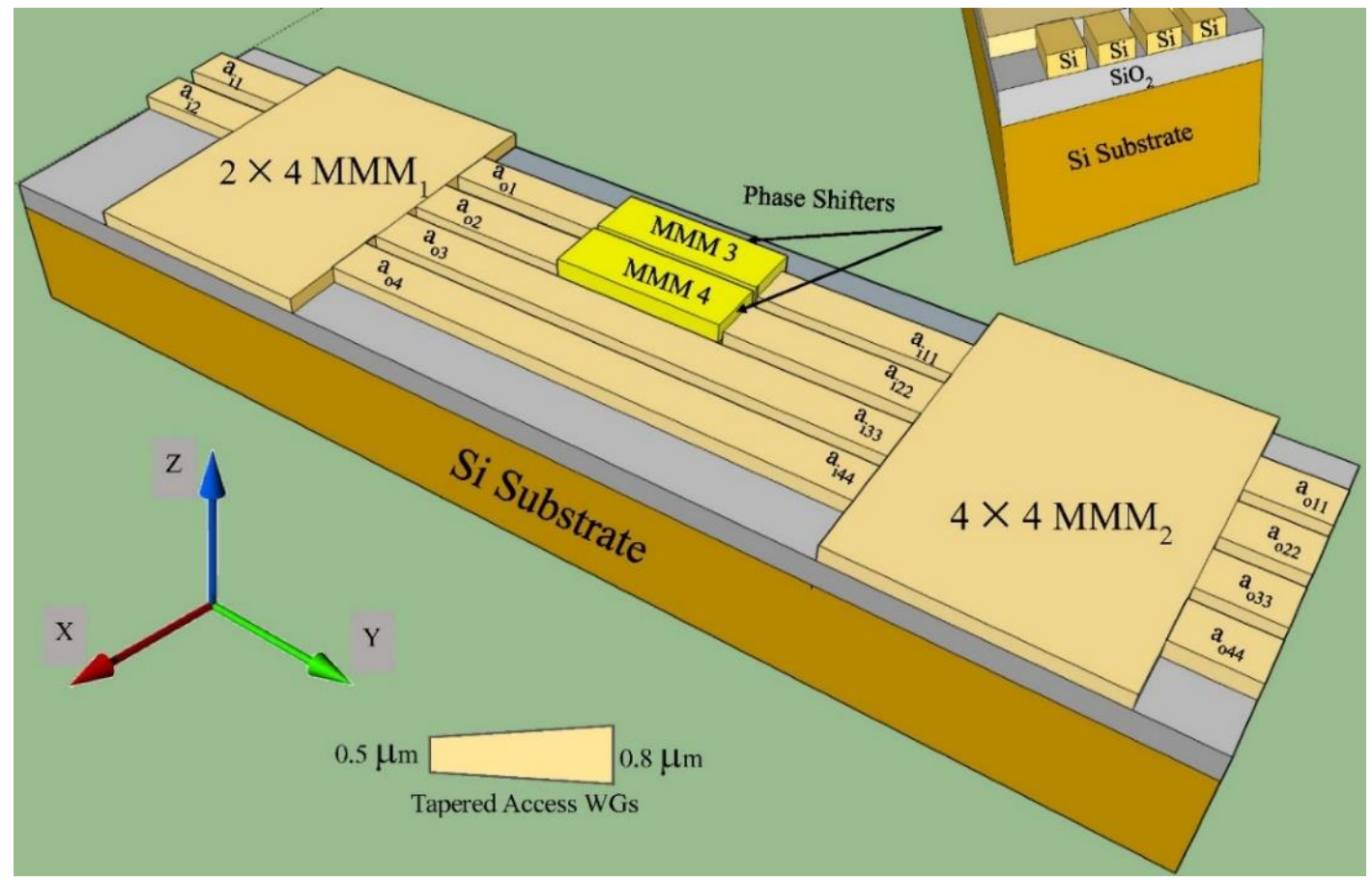

Fig 2. Schematic diagram of the proposed device. In the inset shown are cross-sectional view (top right) and the tapper access waveguide (bottom): $\mathrm{Si}$ substrate $=1000 \mu \mathrm{m}, \mathrm{SiO}_{2}=1000 \mu \mathrm{m}, \mathrm{Si}$ WGs, height $=0.22 \mu \mathrm{m}$, and width $=0.5 \mu \mathrm{m}$. Widths of the substrate and $\mathrm{SiO}_{2}$ layer are constant at $6 \mu \mathrm{m}$. The top layer is Air. In the top inset shown is cross-sectional geometry of the MMI waveguide and in the bottom shown is the tapered waveguide with $0.5 \mu \mathrm{m}$ width in the input and $0.8 \mu \mathrm{m}$ width at the output side, to minimize propagation loss.

To ensure single mode operation of the access waveguides (see, Fig 2 at the bottom), geometrical parameters were derived using 3-dimensional eigenmode expansion method [31]. Air, as the top layer and waveguide height of $0.22 \mu \mathrm{m}$ was chosen for this purpose while the waveguide width was estimated using optical mode solver and FDTD simulation run.

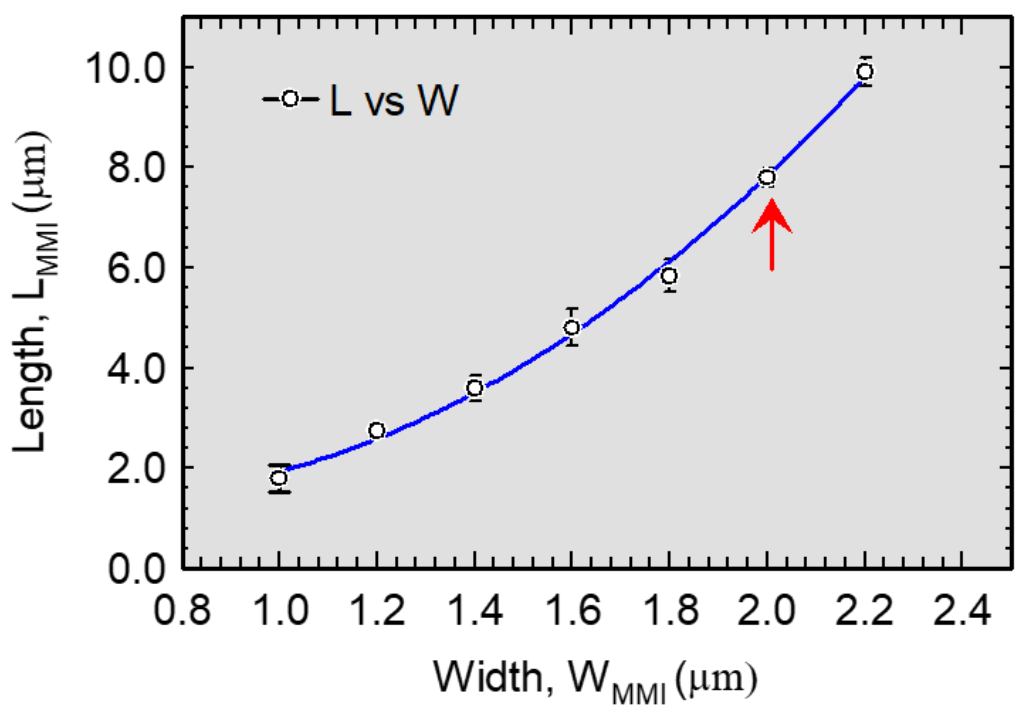

Fig 3: The length of the MMI $\mathrm{MM}_{3,4}$ LMmI, obtained from FDTD simulation, used as a shifter and corresponding waveguide width to obtain single self-image at the output port of the input light. Error bars are within $\pm 1 \%$.

Fig. 3 shows the calculated width of the $\mathrm{MMI}_{3}$ and $\mathrm{MMI}_{4}$, which are designed as a $1 \times 1$ phase shifter, in this case, corresponding to the MMI lengths to form a single image in the output. 
The length of the input and output tapers were varied from 0 to $10 \mu \mathrm{m}$. The width of the $\mathrm{MMI}_{3}$ and $\mathrm{MMI}_{4}$ have been estimated to be $2.0 \mu \mathrm{m}$ and their length has been $8 \mu \mathrm{m}$. The length of the MMI phaseshifter is chosen in such a way that it can support a maximum of 3 optical modes. However, the width should be kept to minimal to avoid cross-talk between the adjacent MMIs, $2 \mu \mathrm{m}$ in this case. The dimension of the phase shifter arm is calculated using MATLAB Mode solver and compared with the dimension obtained from the FDTD simulation tool.

The transmission characteristics of the MMI coupler can be determined using either the self-imaging theory or numerical methods such as the eigenmode method and beam probe method. This work employed two-dimensional FDTD numerical method instead to optimize and verify the designs of MMIs [32] as it was shown to produce sufficiently accurate results in simulating devices based on SOI channel waveguides. Device design for fabrication purpose was carried out using DW-2000 tool accessed through CMC Microsystems [33]. The grid-size used during FDTD simulation was $5 \mathrm{~nm}$ for a waveguide of $0.5 \mu \mathrm{m}$ of width and $0.22 \mu \mathrm{m}$ of thickness. In analogy to reference [19], two mesh override regions were used. The device was the sent to IMEC Belgium for fabrication, and the measurement was performed at the Nano-Optics Laboratory at the Department of Electrical and Computer Engineering, the University of British Columbia.

\subsection{Thermo-optic Heaters}

Change in refractive index along the length of an optical waveguide leads to change in mode propagation constant of the optical signal. Refractive index in a Si waveguide can be tuned using two effects: free career plasma dispersion and thermo-optical effect, similarly to the ones we reported earlier [19]. The thermo-optical effect is relatively stronger as $\mathrm{Si}$ exhibits a strong thermo-optical coefficient of $\mathrm{dn} / \mathrm{dT}=-1.8 \times 10^{-4} / \mathrm{K}$ at $300 \mathrm{~K}$.

In many circumstances, the phase shifting is an essential characteristic of the optical signal as it traverses through waveguides, including switches and filters. Not all kind of phase shifting is, however, useful for switching application. In most cases, the desired phase shift requires special device design consideration. In the present work, two $1 \times 1 \mathrm{MMI}$ devices $\left(\mathrm{MMI}_{3}\right.$ and $\left.\mathrm{MMI}_{4}\right)$ are considered for phase shifting purpose. These are introduced between $\mathrm{MMI}_{1}$ and $\mathrm{MMI}_{2}$ with the aim of tuning/varying the coupling coefficient at the output ports.

In this work, phase shifting and tuning of the phase has been achieved using the thermo-optical effect. A thermal heating element/pad is incorporated into $\mathrm{MMM}_{3}$ and $\mathrm{MMM}_{4}$ to realize the desired phase shifting.

The design and realization of the thermo-optical switch from Si-based MMI require several practical considerations. Examination of the optical field and mode propagation profiles in the slab waveguide is taken as a first step. Using guided mode theory and FDTD tool, the loss of heat in the heater was estimated, and $\mathrm{Al}$ is used in this case.

The operating principle of the thermo-optical switch is based on the change of mode propagation constant of the beam as it propagates through the phase-shifter / modulating arm with respect to the reference arm. This change causes phase difference between the light beam propagating through the phase modulating arm and reference arm and it is given by $\Delta \phi=2 \pi / \lambda(\Delta$ neff $\times$ LMMI), where $\lambda$ is the operating wavelength, $1550 \mathrm{~nm}$ in this case, $\Delta$ neff is the change in refractive index, and Lмм is the length of the modulating arm. Various parameters of the waveguides and heater element are given in Table 2.

Table 2: Physical properties of the material for the heater element

\begin{tabular}{|l|l|l|l|}
\hline $\mathrm{SN}$ & Material & Thermal conductivity $(\mathrm{W} / \mathrm{m} . \mathrm{K})$ & Heating coefficient $(/ \mathrm{K})$ \\
\hline 1 & $\mathrm{Si}$ & 163.25 & $160 \times 10^{-06}$ \\
\hline 2 & $\mathrm{SiO}_{2}$ & 1.405 & $8 \times 10^{-06}$ \\
\hline 3 & $\mathrm{Al}$ & 204 & $24 \times 10^{-06}$ \\
\hline
\end{tabular}

For devices with equal length of the phase modulating/shifter and reference arms such as the one considered in this case, a phase shift of $\pi$ can be introduced with the help of a heater in the modulating 
arm as the refractive index is temperature dependent as $\Delta \phi=2 \pi / \lambda(\mathrm{dn} / \mathrm{dt}) \times \Delta \mathrm{T} \times \mathrm{LmMI}(1+\alpha \Delta \mathrm{T})$, where $\mathrm{dn} / \mathrm{dt}$ is the thermo-optical coefficient $\left(1.86 \times 10^{-4} \mathrm{~K}^{-1}\right.$ for $\left.\mathrm{Si}\right), \lambda$ is the operating wavelength, 1550 $\mathrm{nm}$, and $\alpha$ is the thermal expansion coefficient of $\mathrm{Si}\left(3.6 \times 10^{-6} / 0 \mathrm{~K}\right)$. A device design based on this mathematical formalism acts as a thermo-optical switch and using this formula it is evident that a $\Delta \mathrm{T}$ of $26.77^{\circ} \mathrm{C}$ is required to achieve a phase shift of $\pi$ for a modulating arm length of $8 \mu \mathrm{m}$. The length of the modulating arm needs to be doubling to achieve a phase difference of $\pi / 2$.

An electro-thermal joule heating was considered and an element consisting of $\mathrm{Al}$ as the electrode was examined. Al was chosen as a heating element because of its large thermal conductivity of $204 \mathrm{~W} / \mathrm{m}$ ${ }^{0} \mathrm{C}$ at room temperature. The electrode size regarding the heating area was chosen to be $2 \mu \mathrm{m} \times 8 \mu \mathrm{m}$ to match for the size of the modulating arm. The calculated switching voltage of $3.2 \mathrm{~V}$ corresponds to the average maximum heating temperature of $50-52^{\circ} \mathrm{C}$ as a phase shift of $\pi$ is introduced through the heating pad in the modulating arm with respect to reference arm. For additional information on the theory and calculation of thermo-optic effect based heaters, we refer the interested readers to our earlier paper [19].

To minimize heat loss and spreading of heat to reference arm and to other parts, a trench surrounds the modulating arm, similarly to our earlier works (see Refs $[19,34,35]$ ). The implementation of this design significantly helps prevent heat spreading and loss by over $90 \%$ as the heat required to drive the switch is directly linked to the device size.

\section{Results and discussions}

\subsection{Power distribution characteristics}

Figure 4 shows the power splitting ratio plotted against the optical wavelength (1480-1580 nm). As shown, the power splitting ratio is less sensitive at the wavelength range of $1489-1510 \mathrm{~nm}$, but it became sensitive in the wavelength domain of 1510-1580 $\mathrm{nm}$.

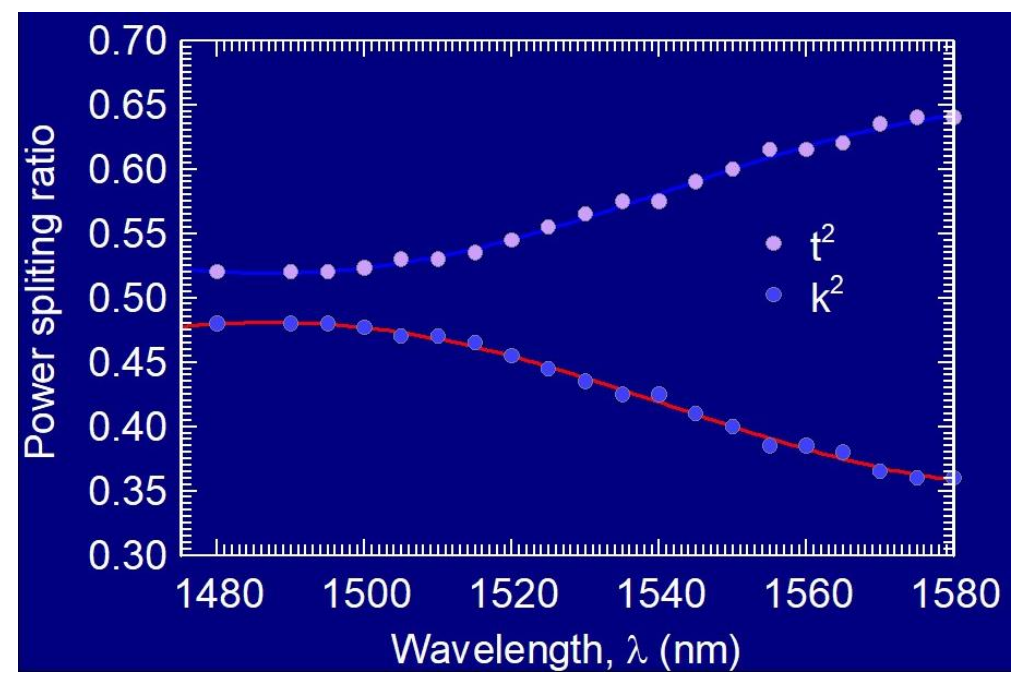

Figure 4: Power splitting ratio of MMIs plotted against the wavelength: bar $\left(\mathrm{t}^{2}\right)$ and crosstalk $\left(\mathrm{k}^{2}\right)$

The normalized power of the optical beam propagating through the MMI is analyzed using the FDTD simulation tool. Fig 4 shows the distribution profile of the optical beam propagating along the $\mathrm{MMI}_{1}$ and $\mathrm{MMI}_{2}$. From this profile, the power splitting ratio at the output ports is $38 \%$ and $61 \%$, respectively at $\lambda$ around $1550 \mathrm{~nm}$.

The optical field profile of the proposed MMI1 is given in Fig. 5 (a) when the incident power is fed from port 1 (ai1). As can be seen, for the given configuration, the optical field is tightly confined at the center of the waveguide with single guided mode propagation and these field profiles suggest that the optical field is not lost through the walls of the waveguide. These field profiles further indicate that there is a room for reducing device width by at least $10 \%$. 


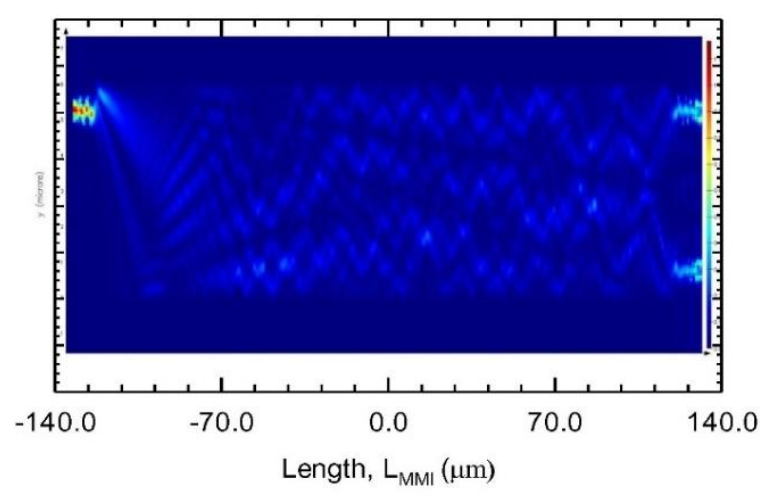

(a)

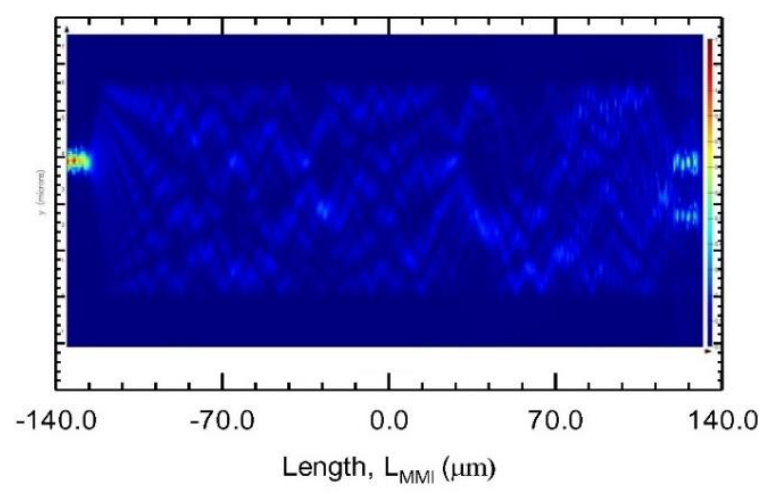

(b)

Fig. 5 Electric field inside the MMI1 (a) input fed through port 1 (b) MMI2 for the input fed through port 2

These field profiles further suggest that the optical beam emerges from the output ports with a power splitting ratio of $0.48 / 0.47$, which closely agrees with our design consideration. Note that the waveguide length was optimized to be $140 \mu \mathrm{m}$ to achieve this ratio of power splitting, as the power splitting ratio and waveguide lengths are related to waveguide width and refractive index.

Figure 5(b) shows the field profile of the $\mathrm{MMI}_{2}$ (Note that LммI2 = LммI1) when the input incident power is sent from Input port 2 (aiz2). In this case, the output power is split into 0.46/0.45 ratio.

The excess loss, which is given by $-10 \times \log _{10}\left(\mathrm{P}_{\text {out }} / \mathrm{P}_{\mathrm{in}}\right)$, and power imbalances, which is expressed as, $-10 \times \log _{10}\left(\mathrm{P}_{\min } / \mathrm{P}_{\max }\right)$, in both cases, are estimated to be $0.65 \mathrm{~dB}$ and $0.09 \mathrm{~dB}$, respectively.

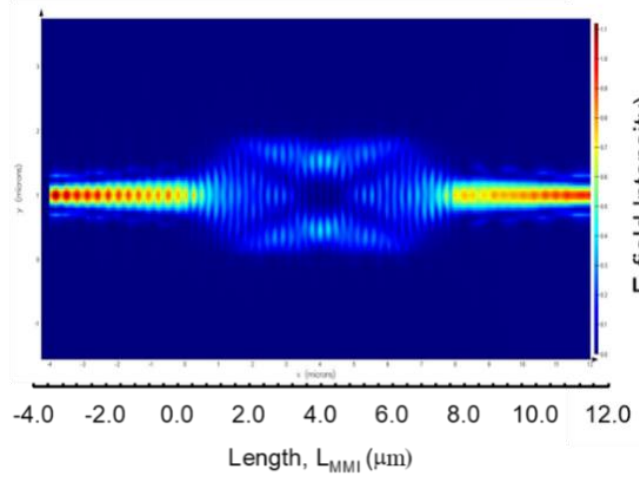

(a)

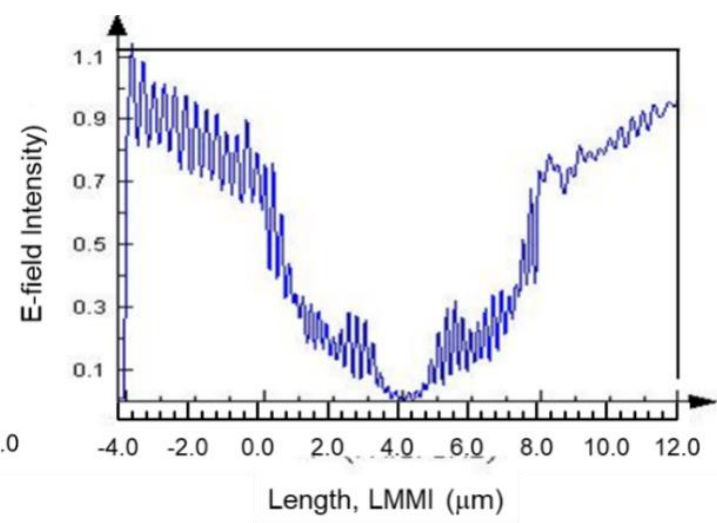

(b)

Fig 6 (a) Field profile of $2 \mu \mathrm{m}$ wide and $8 \mu \mathrm{m}$ long $1 \times 1 \mathrm{MMI}_{3}$ phase shifter without a heater (b) Intensity profile of the $1 \times 1 \mathrm{MMI}_{3}$ phase shifter as a function of its length for a waveguide width, $\mathrm{W}_{\mathrm{MMI}}=2 \mu \mathrm{m}$. The $4 \mu \mathrm{m}$ long tapered access waveguides are connected at both ends of the MMIs to reduce power losses during optical coupling.

The optical field profile of MMI 3 is shown in Fig. 6 (a). These profiles also suggest that the optical wave is tightly confined to the center of the waveguide with single-mode propagation and with no noticeable loss through side walls. This field profile suggests that the width of this waveguide can be reduced by up to $10 \%$. Similarly, to Fig 6(a), the field profile of the light in the linking arms $\mathrm{MMI}_{4}$ showed tightly confined optical filed profile with single-mode propagation and with no seen loss through walls. This is not shown here for clarity.

For the phase shifter, as shown in Fig 10(a), the output to input power ratio is 0.9 to 1 , which means, there is a $10 \%$ loss of power. The value suggests that there is destructive interference in the middle of the phase shifter. The output power gradually builds up, and at the exit, it goes up to $90 \%$. 
The intensity profile/power profile of the phase-shifter arm as a function of the Lмміз is given in Fig 6(b). As shown in it, the power coupling ratio can be tuned/changed by varying the phase shift at the phase modulating arm.

The optical device/switching characteristics of the system was considered and analyzed by connecting all four components of the device; namely; $\mathrm{MMI}_{1}, \mathrm{MMI}_{2}, \mathrm{MMI}_{3}$ and $\mathrm{MMI}_{4}$ (see Fig. 1), with $\mathrm{MMI}_{1}, \mathrm{MMI}_{2}$ each with $\mathrm{LMMI}=3 \times \mathrm{L}_{\pi} / 2$ and $\mathrm{MMI}_{3} / \mathrm{MMI}_{4}$ and access waveguides with total length of $16 \mu \mathrm{m}$ each. As shown, the coupling coefficients were varied using Al heating pad in the phase shifter linking arms. Similarly to [19], a trench is created around the heating pad to prevent heating loss.

\subsubsection{Case I: $\Delta \varphi=0$ (Heating disabled)}

For the light input at port ail, preliminary simulation results suggested that the normalized output powers from port 1 to 4 are $0,0,0,0.75$, respectively when phase shifting is not considered. The excess loss, in this case, is estimated to be $0.95 \mathrm{~dB}$, and the imbalance of power is estimated to be $0.1 \mathrm{~dB}$.

\subsubsection{Case II: $\Delta \varphi=\pi / 2$ (Heating enabled)}

Upon the introduction of $\pi / 2$ phase shift in the first phase shifter, i.e., $\mathrm{MMI}_{3}$, the normalized output power at the output ports is estimated to be $0.45,0,0,0.44$, respectively. For the light input at port ai2, the scenario is different when a phase shift of $\pi / 2$ is introduced in the second phase shifter, i.e., $\mathrm{MMI}_{4}$ : The normalized output powers, in this case are estimated to be $0.75,0,0,0.25$, respectively. The excess loss and power imbalances were estimated to be $0.90 \mathrm{~dB}$ and $0.20 \mathrm{~dB}$, respectively.

The calculated power transmission coefficients of the $\mathrm{MMI}_{1}$ and $\mathrm{MMI}_{2}$ couplers are $\left|\gamma_{1}\right|^{2}=0.75$ and $\left|\gamma_{2}\right|^{2}=0.45$. The power transmission coefficients of both these couplers can be adjusted using the phase shifter arms MMIs and MMI4.

The insertion loss of the MMI coupler/splitter is given by $L_{\text {ins }}(\mathrm{dB})=-10 \log _{10}\left[\mathrm{P}_{\text {out }} / \mathrm{P}_{\text {in }}\right]$, where $\mathrm{P}_{\text {in }}$ is power input in the input port and Pout is power output at one of the output ports (either bar or cross ports). For the input power of $1 \mathrm{~mW}$, the insertion loss for all the four output ports was estimated to be around $0.9 \mathrm{~dB}$ for each port. These values suggest that the power transferred energy from the input port is transferred through the devices almost without any significant loss, suggesting the fact that the device is energetically viable and efficient.

\section{$4.2 \quad$ Switching Characteristics}

Figure 7 shows a plot of output power state as a function of heating power to switch the output state from 0 to 1 or vice versa. From the heating power of $1.4 \mathrm{~mW}$ and resistivity of $\operatorname{Si}\left(2.3 \times 10^{3} \Omega . \mathrm{m}\right)$, the switching voltage required is $1.79 \mathrm{~V}$.

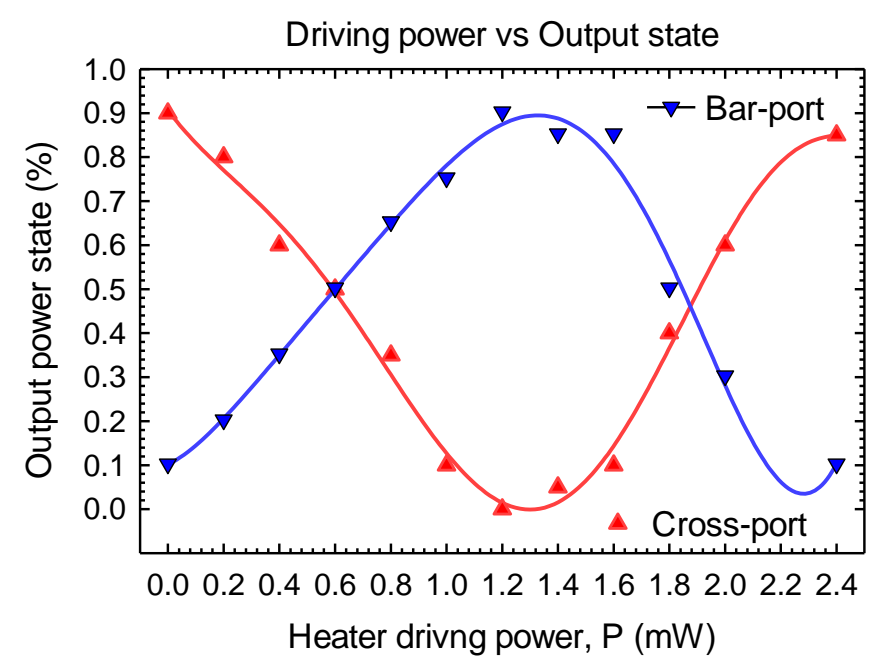

Fig. 7 Output power vs driving power at the cross and bar ports of the MMI without trenches. 
The switching parameters can be further reduced by optimizing the surface area, length, and thickness of the heating pad. The reduction in power loss is almost by a factor of 2 as compared to the conventional phase shifter without the trench [36]. These results suggested that the total device length should be chosen to be $324 \mu \mathrm{m}$ to achieve $3 \mathrm{~dB}$ operation. This result is comparable and consistent with the result estimated using self-imaging theory [6].

\section{Conclusions.}

We proposed an optical switch based on 2x4 multimode interference on SOI technology and adopted a new approach for low-loss heaters. The device showed output power splitting ratio of $0.48 / 0.46$ or $0.75 / 0.25$ as obtained by simulation. The importance of this device is that it can act as two independently operating digital switches with improved efficiency and the low footprint $(6 \mu \mathrm{m} \times 324$ $\mu \mathrm{m})$. The device showed a small insertion loss of around $0.9 \mathrm{~dB}$ for each port, a small heating power of $1.4 \mathrm{~mW}$, and a switching voltage of around $1.79 \mathrm{~V}$. Creating a trench around the Al heating pad and isolating the heater has been shown to be critical in reducing the spreading of power into surrounding regions and power loss in these devices [19]. In addition to optical switches, the device has a potential to be used for optical interconnects, micro-ring resonator applications as well as highly sensitive bio-sensors that can be used to detect various types of samples such as poisonous gas media to bio-samples, environmental monitoring, and imaging.

Acknowledgments: Planning and design work was conducted at the University of British Columbia under the guidance of Drs. Nicolas A. F. Jaeger and Lukas Chrostowski. Authors acknowledge valuable feedback and critical comments during the planning and execution of this work. Authors thank Dan Deptuck at CMC Microsystems [33] for providing technical support during design, verification, and fabrication process. Authors thank Elizabeth Rizal, a visual-arts student at York University, Canada, for sketching Fig. 2 for the manuscript.

Author Contributions: B.N and C.R. conceived and designed the experiments; B.N. and C.R performed the experiments; C.R. and B.N. analyzed the data; CR analyzed the design and verified the correctness of the design. B.N. wrote the paper, and all the authors contributed equally.

Conflicts of Interest: The authors declare no conflict of interest.

References

1. Chrostowski, L.; Hochberg, M. Silicon Photonics Design: From Devices to Systems; Cambridge University Press: 2015.

2. For EpiPhotonics, $\quad$ Retrieved from http://www.epiphotonics.com/pdfs/EpiPhotonics Products.pdf. Availabe online: (accessed on May 15).

3. Gautam, R. Silicon microring resonator loaded Mach-Zehnder interferometer for low power optical modulation, switching and bio-sensing. Yokohama National University, Japan, 2014.

4. Lu, L.; Zhou, L.; Li, S.; Li, Z.; Li, X.; Chen, J. $4 \times 4$ nonblocking silicon thermo-optic switches based on multimode interferometers. Journal of Lightwave Technology 2015, 33, 857-864.

5. Boeck, R.; Caverley, M.; Chrostowski, L.; Jaeger, N.A.F. Experimental demonstration of a silicon-on-insulator high-performance double microring filter using MZI-based coupling. Optics Letters 2015, 40, 276-279.

6. Bryngdahl, O. Image formation using self-imaging techniques. JOSA 1973, 63, 416-419.

7. Le, T.-T. Two-channel highly sensitive sensors based on $4 \times 4$ multimode interference couplers. Photonic Sensors 2017, 7, 357-364.

8. Rabus, D.G. Integrated ring resonators; Springer: 2007. 
9. Pitilakis, A.; Kriezis, E.E. Longitudinal $2 \times 2$ Switching Configurations Based on ThermoOptically Addressed Dielectric-Loaded Plasmonic Waveguides. Journal of Lightwave Technology 2011, 29, 2636-2646.

10. Zinke, T.; Fischer, U.; Schueppert, B.; Petermann, K. Theoretical and experimental investigation of optical couplers in SOI. In Proceedings of Photonics West '97; p. 10.

11. Ota, M.; Sumimura, A.; Fukuhara, M.; Ishii, Y.; Fukuda, M. Plasmonic-multimodeinterference-based logic circuit with simple phase adjustment. Scientific reports 2016, 6, 24546.

12. Oulton, R.F.; Sorger, V.J.; Genov, D.; Pile, D.; Zhang, X. A hybrid plasmonic waveguide for subwavelength confinement and long-range propagation. nature photonics 2008, 2, 496.

13. Xing, J.; Li, Z.; Yu, Y.; Yu, J. Low cross-talk $2 \times 2$ silicon electro-optic switch matrix with a double-gate configuration. Optics letters 2013, 38, 4774-4776.

14. Xie, N.; Hashimoto, T.; Utaka, K. Design and performance of low-power, high-speed, polarization-independent and wideband polymer buried-channel waveguide thermo-optic switches. Journal of Lightwave Technology 2014, 32, 3067-3073.

15. Suzuki, K.; Cong, G.; Tanizawa, K.; Kim, S.-H.; Ikeda, K.; Namiki, S.; Kawashima, H. Multiport optical switches integrated on Si photonics platform. IEICE Electronics Express 2014, 11, 20142011-20142011.

16. Lagali, N.S.; Paiam, M.R.; MacDonald, R.I.; Driessen, A. Analysis of generalized MachZehnder interferometers for variable-ratio power splitting and optimized switching. Journal of lightwave technology 1999, 17, 2542.

17. Morrissey, P.; Yang, H.; Sheehan, R.; Corbett, B.; Peters, F. Design and fabrication tolerance analysis of multimode interference couplers. Optics Communications 2015, 340, $26-32$.

18. Li, Z.; Zhou, L.; Lu, L.; Zhao, S.; Li, D.; Chen, J. $4 \times 4$ nonblocking optical switch fabric based on cascaded multimode interferometers. Photonics Research 2016, 4, 21-26.

19. Rizal, C.; Niraula, B. Compact Si-based asymmetric MZI waveguide on SOI as a thermooptical switch. Optics Communications 2018, 410, 947-955.

20. Bryngdahl, O. Self-imaging with an optical tunnel for image formation. Google Patents: 1974.

21. Heaton, J.M.; Jenkins, R.M. General matrix theory of self-imaging in multimode interference (MMI) couplers. IEEE Photonics Technology Letters 1999, 11, 212-214.

22. Bogaerts, W.; Fiers, M.; Dumon, P. Design challenges in silicon photonics. Selected Topics in Quantum Electronics, IEEE Journal of 2014, 20, 1-8.

23. Hamada, H. Theoretical time-domain study of self-imaging properties in a multimode interference coupler. Applied optics 2007, 46, 3843-3849.

24. He, S.; Ao, X.; Romanov, V. General properties of $\mathrm{N} \times \mathrm{M}$ self-images in a strongly confined rectangular waveguide. Applied optics 2003, 42, 4855-4859.

25. Soldano, L.B.; Pennings, E.C. Optical multi-mode interference devices based on self-imaging: principles and applications. Journal of lightwave technology 1995, 13, 615-627.

26. Berry, G.; Burke, S. Analysis of optical rib self-imaging multimode interference (MMI) waveguide devices using the discrete spectral index method. Optical and quantum electronics 1995, 27, 921-934.

27. Bachmann, M.; Besse, P.; Melchior, H. Overlapping-image multimode interference couplers with a reduced number of self-images for uniform and nonuniform power splitting. Applied optics 1995, 34, 6898-6910. 
28. Pennings, E.; Deri, R.; Scherer, A.; Bhat, R.; Hayes, T.; Andreadakis, N.; Smit, M.; Soldano, L.; Hawkins, R. Ultracompact, low-loss directional couplers on $\mathrm{InP}$ based on self-imaging by multimode interference. Applied physics letters 1991, 59, 1926-1928.

29. Ulrich, R.; Ankele, G. Self-imaging in homogeneous planar optical waveguides. Applied Physics Letters 1975, 27, 337-339.

30. Latunde-Dada, K.A.; Payne, F.P. Theory and design of adiabatically tapered multimode interference couplers. Journal of lightwave technology 2007, 25, 834-839.

31. Sudbo, A.S. Film mode matching: a versatile numerical method for vector mode field calculations in dielectric waveguides. Pure and Applied Optics: Journal of the European Optical Society Part A 1993, 2, 211.

32. Sullivan, D.M.; Mossman, S.; Kuzyk, M.G. Time-Domain Simulation of Three Dimensional Quantum Wires. PloS one 2016, 11, e0153802.

33. CMC Microsystem. Nano Silicon-on-Isulator Process Technology. Availabe online: http://www.cmc.ca/ (accessed on

34. Pokharel, R.K.; Rizal, C.S. Design and characterization of a compact optical device based on Mach-zehnder interferometer in Silicon-on-insulator technology. In Proceedings of 15th Annual Meetting of the Pacific Center for Advanced Materials (PCAMM), Vancouver, Canada, August 15, 2011.

35. Rizal, C.; Niraula, B. Nanoscale Silicon Waveguide Based Thermo-Optic Sensor Using a Compact Mach-Zehnder Interferometer. MDPI Photonics 2016, 2016080151, 19, doi:doi: 10.20944/preprints201608.0151.v1.

36. Al-hetar, A.M.; Yulianti, I.; Supa'at, A.S.M.; Mohammad, A. Thermo-optic multimode interference switches with air and silicon trenches. Optics Communications 2008, 281, 4653 4657. 